\title{
Extended scaffold glucuronides: en route to universal synthesis of O-aryl glucuronide prodrugs
}

\author{
Raoul Walther, ${ }^{a}$ Morten T. Jarlstad Olesen, ${ }^{\mathrm{b}}$ Alexander N. Zelikin ${ }^{\mathrm{a}, \mathrm{b}} *$
}

\footnotetext{
a. Department of Chemistry, Aarhus University, Aarhus, Denmark

b. iNano Interdisciplinary Nanoscience Centre, Aarhus University, Aarhus, Denmark E-mail: zelikin@chem.au.dk.
}

\begin{abstract}
We demonstrate that extended scaffold based on a self-immolative linker (SIL) enables universal production of 0 -aryl glucuronide prodrugs: high yield glucuronidation is performed on a precursor substrate (SIL) and the subsequent drug conjugation proceeds via less challenging chemical reactions.
\end{abstract}

Targeted drug delivery is fast becoming the mainstream of biomedicine and is expected to revolutionize the treatment of diverse diseases. Drug targeting can be achieved using antibody-drug conjugates (ADC), ${ }^{1-3}$ via the organ-specific activation of prodrugs, ${ }^{4}$ "enzyme-prodrug therapies" (EPT), ${ }^{5}$ or localized drug synthesis mediated by the diseased tissue itself. ${ }^{6}$ In each of these methodologies, key to success is the nature of the prodrug. It has to be biocompatible and preferably devoid of pharmacological activity, yet become a potent therapeutic upon bioconversion. Of all the prodrugs applied in the EPT and the tumor-activated drug syntheses, glucuronides stand out as the privileged scaffold. This is due to the minimized cell entry of glucuronides, which affords a high ratio between the toxicityrelated I $\mathrm{C}_{50}$ for the prodrug and the drug. ${ }^{4}$ Glucuronides are also advantaged in that these typically afford a significantly enhanced aqueous solubility, a feature of high importance for hydrophobic drugs. However, disadvantage of the glucuronides lies in the synthetic hurdles for their synthesis. ${ }^{7,} 8$ In nature, glucuronides are formed enzymatically during the Phase II metabolism in the liver. In the lab, glucuronide syntheses are carried out via multi-step, laborious routes involving protective groups. Furthermore, chemical glucuronidation lacks a universal methodology and glucuronidation requires tedious optimization for each specific drug, even within the same class of molecules (e.g. phenols). 9,10

In this work, we specifically address this challenge and develop a modular methodology for the synthesis of glucuronide prodrugs, based on the extended scaffold glucuronides that in their structure include a self-immolative linker (SIL). ${ }^{11}$ We employ SIL such that the most synthetically challenging, glucuronidation step becomes universal, whereas drug conjugation is achieved via less demanding reactions. We investigate this on the example of $O$-aryl glucuronides and demonstrate that SIL serves to resolve the synthetic hurdles of glucuronidation and affords straightforward, good yield syntheses of diverse extended scaffold glucuronides. We then compare the natural vs extended scaffold glucuronides in terms of kinetics of drug recovery as well as their performance in an in
A Direct glucuronidation

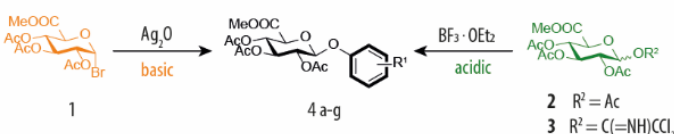

B Extended scaffold
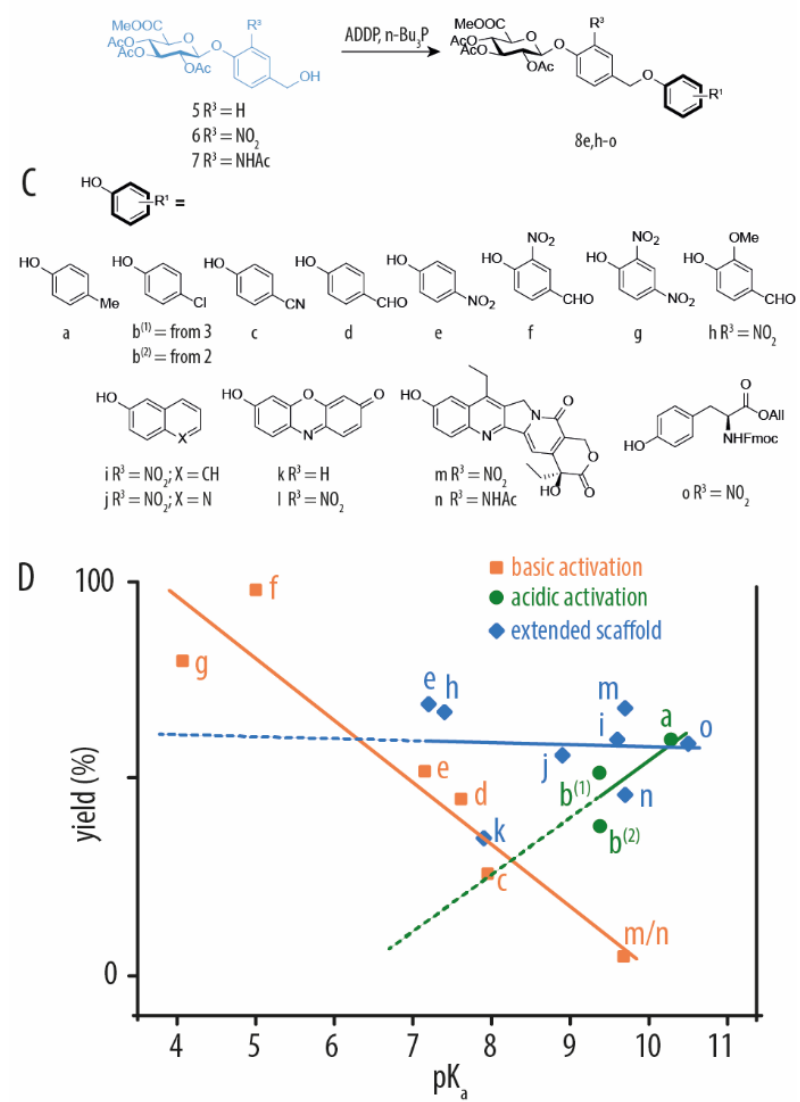

Figure 1. (A) Schematic representation of existing methodologies to O-aryl glucuronidation and (B) the proposed herein "extended scaffold" approach towards establishing a universal glucuronidation method; (C) chemical formulas of the phenolic compounds tested in this work as substrates for traditional and extended scaffold glucuronidation; (D) Scatter plot representation of the scope and limitations of the traditional O-aryl glycosylation methods, with the reaction yield greatly dependent on the pKa of the phenol, and the proposed herein extended scaffold glucuronidation method in which case glucuronidation yields are virtually constant across the range of pKa of the phenols. 
vitro EPT. Results of this study are significant in establishing a modular glucuronidation platform to synthesize these privileged prodrugs for diverse drug targeting applications.

First, to verify the scope and limitations of "classical" chemical glucuronidation, we investigated the Koenigs-Knorr coupling9, 10 of phenols with glucuronyl bromide $\mathbf{1}^{12}$ (Figure $1 \mathrm{~A}$ ). These reactions yielded the $O$-aryl glucuronides with excellent $\beta$ selectivity but glycosylation yields were greatly dependent on the $\mathrm{p} K_{\mathrm{a}}$ of the phenol (Figure 2, for numerical values of yields, see Table S1) and became near zero for $p$-chlorophenol and analogues with a higher $\mathrm{pKa}$. Our efforts to optimize the Koenigs-Knorr-based glucuronidation through the variation of the solvent, the promoters (including $\mathrm{Ag}_{2} \mathrm{CO}_{3}$ and $\mathrm{AgOTf}$ ), and the additives were met with failure (Table S2): instead of improved glycosylation outcome we observed formation of the common glycal or orthoester as side products. We also considered that glucuronidation of the electron-rich phenols can be performed under the Lewis acid catalyzed conditions (using the anomeric acetate $\mathbf{2}^{13}$ or trichloroacetimidate $\mathbf{3}^{14}, 15$ ). Yet these too are not universal since phenols are ambident nucleophiles and under Lewis acid conditions likely afford $C$ glycosides. ${ }^{16,17}$ Based on these results, as well as the extensive prior art on this subject, we conclude that a "universal" glucuronidation pathway may not be identified through the variation of glycosylation conditions. Instead, we propose that such a synthetic opportunity can be engineered using a cunning tool of chemistry, namely SILs.

Historically, SILs have been developed to optimize the triggered drug release by virtue of increasing accessibility of the scissile bond to the enzyme for bioconversion. ${ }^{18}$ In recent years, we and others also recognized the power of SIL to enable "un-natural" chemistry within the existing molecules, such as the use of thioldisulfide chemistry in thiol-free molecules. ${ }^{19}$ Common SIL include $p$-hydroxylbenzyl alcohol PHBA and its close analogues, $p$-aminobenzyl alcohol ${ }^{20}$ and $p$-mercaptobenzyl alcohol, ${ }^{21}$ which are well-established tools of medicinal chemistry, most notably as spacers for the enzyme-activated prodrug decomposition. In this work, we propose to use PHBA as a unique tool for the design of $O$-aryl glucuronides. The vision put forward is that in this case, the most synthetically challenging, glucuronidation step, becomes universal whereas drug conjugation is achieved via less demanding reactions.

The synthesis of $O$-aryl glucuronide was accomplished using 4hydroxylbenzaldehyde and the o-nitro analogue hereof (phenols $\mathbf{d}$ and $\mathbf{f}$, Figure 1). The two molecules exhibited high yields of glycosylation (Figure 2, Table S1) and were conveniently reduced to the corresponding benzyl alcohols (5, 6) with near-quantitative yields. The nitro-group may be preserved in the structure of the prodrug or, to avoid the risk of immune reactions, ${ }^{22}$ can be reduced at this stage to the corresponding amine and optionally acetylated 7 (Figure 1, Figure S1). The latter opportunity is specifically important with the view of enhancing synthetic possibilities for e.g. bioconjugation of glucuronides to antibodies, ${ }^{23,24}$ albumin, ${ }^{6}$ etc. Conjugation of phenols to the extended glucuronide scaffold was optimized using resorufin as a model turn-on fluorescent probe. Conventional synthesis of the key alkyl-aryl ether bond through the Williamson-ether approach using benzyl halides/mesylates $\mathbf{S 4}$ or under Lewis-acidic conditions through benzyl 2,2,2-trichloroacetimidate intermediate S5 were unsatisfactory (Figure S2). Modified Mitsunobu reaction ${ }^{25}$ on the other hand gave encouraging results (Table S3 entries $a-b$ ), shortened the synthetic sequence, and circumvented the transformation of the benzyl alcohol glucuronide intermediate into a suitable precursor for an $\mathrm{S}_{\mathrm{N}} 2$ reaction. However, it afforded a high yield of the unwanted side product, namely the $\Delta_{4,5}$-dehydro compound. ${ }^{7,} 8$ Nevertheless, under optimized conditions (Table S3), the yield of the $\Delta_{4,5}$-dehydro compound was minimized to ca. $2.5 \%$ whereas formation of the desired product conjugate was maximized to $47 \%$.

Next, we investigated the substrate scope of the optimized reaction conditions (Figures 1D). Derivatives of 4-nitrophenol 8e, 2-naphthol 8i, 6-hydroxyquinoline $\mathbf{8 j}$ and vanillin $\mathbf{8 h}$ were successfully isolated with good yields (50-70\%), already extending well beyond the substrate scope for Koenig-Knorr glucuronidation conditions in terms of the $\mathrm{pKa}$ of the phenol. Furthermore, extended scaffold glucuronide of a tyrosine derivative (80, pKa over 10) was also obtained in good isolated yield paving the way to the prodrugs of biological drugs as well as tubulysin A. Finally, glycosylation product for a marketed anticancer drug SN-38 (with a pKa of the phenol of 9.7) was delivered in a yield of $68 \%$ for the nitro-containing linker $8 \mathrm{~m}$ and $46 \%$ for the acetamide-containing linker $8 n$, and either case being significantly better than the $5 \%$ yield of the direct glucuronidation (Table S1). Taken together, results in Figure 1 illustrate that the proposed synthetic pathway involving (nitro)PHBA SIL as the glucuronidation partner with a subsequent synthesis of aryl-ether delivers a considerable synthetic flexibility and affords glucuronide derivatives for a broad range of phenols (Figure 1). Suitable substrates include phenols across the range of $\mathrm{p} K a$ for the phenol, as high as 10.5 for tyrosine, and accommodate a straightforward synthesis of derivatives for $\mathrm{SN}-38$.

Deprotection conditions were validated for the resorufin turnon fluorescent probes $\mathbf{9}$ and $\mathbf{1 0}$ and the derivatives of SN-38 (11 and 12). For resorufin, deprotection was carried out via Zémplen deacetylation and subsequent saponification of the methyl ester by $2 \mathrm{M} \mathrm{NaOH}$ (Figure S3). For SN-38, saponification was optimized well beyond the reported yields (typically 12$27 \%)^{3,26}$ and using $\mathrm{Ba}(\mathrm{OH})_{2} 8 \mathrm{H}_{2} \mathrm{O}^{27}$ in anhydrous $\mathrm{MeOH}$, prodrugs 11 and 12 were obtained in $86 \%$ and $48 \%$ yield, respectively, with the lactone moiety of $\mathrm{SN}-38$ still in place. (Figure S3).

Next, we aimed to validate if the extended scaffold glucuronides are accepted by the enzyme for bioconversion and if so, quantitate the kinetics of enzymatic catalysis. For the prodrugs of resorufin $(\mathbf{9}, \mathbf{1 0})$ and SN-38 $(\mathbf{1 1}, \mathbf{1 2})$, enzymatic scission of the glycosidic linkage affords a phenolic compound that undergoes a 1,6-elimination with the formation of resorufin or $\mathrm{SN}-38$, respectively (Figure $2 \mathrm{~A}$ ). Resorufin prodrugs were designed as fluorogenic probes, for which the scission of the glucuronide bond and the ensuing self-immolation of PHBA afford resorufin, accompanied by a drastic increase in fluorescence. The latter phenomenon provides a convenient means to monitor and quantify enzymatic conversion of the substrate. Incubation of resorufin- $\beta$-D-glucuronide (reso-GlcA) and the two extended 
A

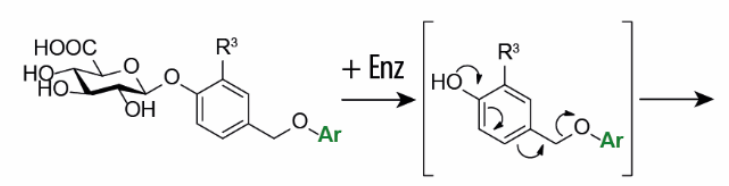

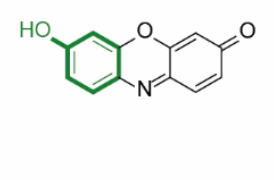

$9 \mathrm{R}^{3}=\mathrm{H} ; \mathrm{Ar}=$ resorufin

$10 \mathrm{R}^{3}=\mathrm{NO}_{2} ; \mathrm{Ar}=$ resorufin

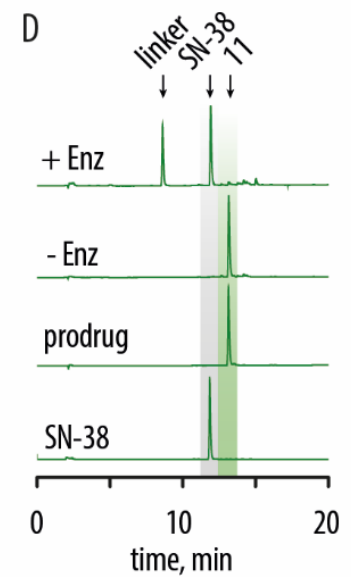

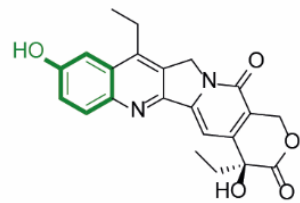

$11 \mathrm{R}^{3}=\mathrm{NO}_{2} ; \mathrm{Ar}=\mathrm{SN}-38$

$12 \mathrm{R}^{3}=\mathrm{NHAC} ; \mathrm{Ar}=\mathrm{SN}-38$
B

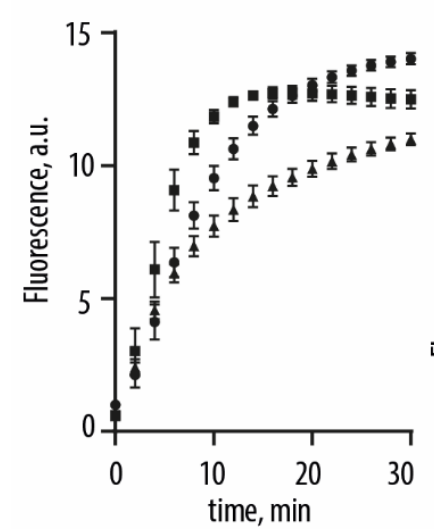

\section{C}

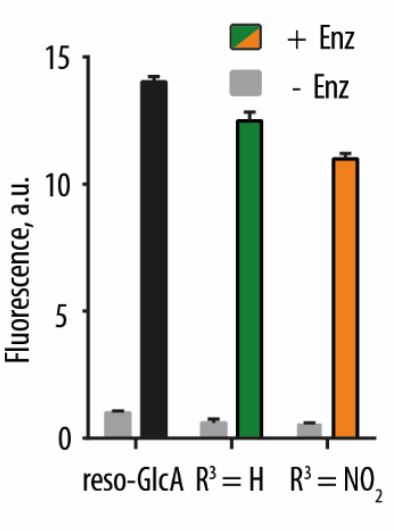

$E$

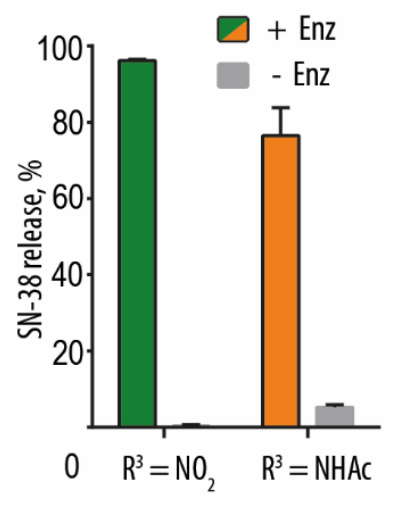

Figure 2. A) Schemtic illustration of the bioconversion and ensuing immolation of the extended scaffold glucuronides; B) Evolution of fluorescence intensity $\left(\lambda_{\mathrm{ex}}=570 \mathrm{~nm} ; \lambda_{\mathrm{em}}=585 \mathrm{~nm}\right.$ ) in solutions of prodrugs reso-GlcA $(\bullet), \mathbf{9}(\boldsymbol{\square})$, and $\mathbf{1 0}(\mathbf{\Lambda})$ at a final concentration of $13 \mu \mathrm{M}$ in PBS in the presence of $1 \mathrm{mg} \mathrm{L}^{-1} \beta$-Glu at $37^{\circ} \mathrm{C}$; C) Fluorescence intensity in solutions of reso-GlcA, $\mathbf{9}$, and $\mathbf{1 0}(13 \mu \mathrm{M}$ in PBS) upon incubation over $2 \mathrm{~h}$ in the presence or absence of $\beta$-Glu $\left(1 \mathrm{mg} \mathrm{L}^{-1}\right)$; D) Representative normalized analytical HPLC traces illustrating bioconversion of the extended scaffold glucuronide of SN-38 (100 $\mu \mathrm{M}$ prodrug, $1 \mathrm{mg} \mathrm{L}^{-1}$ enzyme, PBS, $\left.2 \mathrm{~h}\right)$; E) Quantitative data on the release of SN-38 from its extended scaffold glucuronides (100 $\mu \mathrm{M}$ prodrug, $1 \mathrm{mg} \mathrm{L}^{-1}$ enzyme, PBS, $2 \mathrm{~h}$ ).

scaffold resorufin prodrugs $\mathbf{9}$ and $\mathbf{1 0}$ in the presence of $\beta$-Glu afforded very similar fluorescence evolution profiles (Figure $2 \mathrm{~B}$ ) and levels of total attained fluorescence after $2 \mathrm{~h}$ of enzymatic bioconversion (Figure $3 \mathrm{C}$ ). Fluorescence evolution profiles were treated to estimate the parameters of enzymatic catalysis, namely $K_{\mathrm{m}}$ and $k_{\text {cat }}$, Table 1 . In applying the Michaelis-Menten equations, we assumed that enzymatic bioconversion (not the self-immolation) is the rate limiting step in the overall drug release. $K_{\mathrm{m}}$ values for the extended scaffold prodrugs were similar to those of the native glucuronide with direct linkage, which illustrates that the extended glucuronide scaffold does not result in an inferior prodrug design compared to the natural glucuronide scaffold. Furthermore, incorporation of a nitrosubstituent ortho to the glycosidic linkage (compound 10) drastically lowered the $K_{\mathrm{m}}$ suggesting that electron-withdrawing groups positively modulate enzyme affinities for PHBAcontaining prodrugs, as has been previously observed for other phenyl glycosides. ${ }^{28,} 29$

For the prodrugs of $\mathrm{SN}-38$, the main characterization tool was HPLC (Figure 2D,E). In the presence of $\beta$-Glu, the prodrugs readily released the constituting toxin and this process was near-complete within $2 \mathrm{~h}$, whereas incubation of prodrugs in PBS without the enzyme showed negligible drug release over 24 h (Figure 2D; Figure S4 for 12). We also observed that prodrug bioconversion for $\mathrm{SN}-38$ was accompanied by an increase in fluorescence (Figure S5), a phenomenon lending itself for quantitative kinetics measurements. The $K_{\mathrm{m}}$ values for the two SIL-containing SN-38 prodrugs ( $42 \pm 4$ and $50 \pm 5 \mu \mathrm{M}$ for 11 and 12, respectively, Table 1 ) were similar to the prodrugs of resorufin and the glucuronide prodrugs of campthothecin and analogues. ${ }^{3,} 26$ In turn, the catalytic efficiency was 10 -fold higher than that for resorufin, yet similar for prodrugs $\mathbf{1 1}$ and $\mathbf{1 2}$.

Lastly, we aimed to characterize the native and the extended scaffold glucuronides of SN-38 in cell culture. Anti-proliferative properties of the prodrugs were evaluated in the human cervical cancer HeLa cells (Figure S6 and Table 1). In short, the growth medium was supplemented with prodrugs in a dilution series ranging from $5.1 \times 10^{-12} \mathrm{M}$ to $10 \times 10^{-6} \mathrm{M}$, in the presence or absence of $\beta$-Glu. Without enzymatic activation, the natural metabolite SN-38- $\beta$-D-glucuronide (SN-38-GIcA) and the two glucuronide prodrugs $\mathbf{1 1}$ and $\mathbf{1 2}$ exhibited $\mathrm{IC}_{50}$ values between 0.6 and $2 \mu \mathrm{M}$. In other words, extended scaffold glucuronides were successful in masking toxicity of the incorporated anticancer drug (for $\mathrm{SN}-38, \mathrm{IC}_{50}=5.5-16 \mathrm{nM}$ ). Notably, the least toxic prodrug was the acetylated extended scaffold product 12. Enzymatic bioconversion shifted the $\mathrm{IC}_{50}$ values to 59 and $82 \mathrm{nM}$ for prodrugs 11 and 12, respectively (Table 1 ). Toxicity data reveal that the $\mathrm{QIC}_{50}$ values (i.e. the ratio between nonactivated and activated prodrug in terms of $\mathrm{IC}_{50}$ ) for the extended scaffold glucuronide was somewhat lower than that for the native glucuronide $\left(\mathrm{QIC}_{50}\right.$ of 23 for the acetylated prodrug 12 vs 100 for SN-38-GICA), possibly due to increased hydrophobicity of the linker.

Taken together, results of this study demonstrate that extended scaffold design comprising a self-immolative linker delivers the highly warranted "universal" strategy for the synthesis of glucuronide prodrugs. The SIL linker is used such that the synthetically challenging glucuronidation step is performed in 
Table 1. Michaelis-Menten kinetic parameters and quantitative in vitro cytotoxicity data for the prodrugs of resorufin and $\mathrm{SN}-38$. I $\mathrm{C}_{50}$ values were determined in human cervical cancer HeLa cells over $72 \mathrm{~h}$ cell culture (Figure $2 \mathrm{~F}$ ). $\mathrm{QIC} \mathrm{C}_{50}=I \mathrm{I}_{50}$ (prodrug) $/ \mathrm{IC} \mathrm{C}_{50}$ (prodrug in presence of $\beta-\mathrm{Glu}$ ).

\begin{tabular}{|c|c|c|c|c|c|c|}
\hline entry & $\begin{array}{l}\boldsymbol{K}_{\mathrm{m}} \\
{[\mu \mathrm{M}]}\end{array}$ & $\begin{array}{l}\boldsymbol{k}_{\mathrm{cat}} \\
{\left[\mathrm{s}^{-1}\right]}\end{array}$ & $\begin{array}{l}\boldsymbol{k}_{\mathrm{cat}} / \boldsymbol{K}_{\mathrm{m}} \\
{\left[\mathrm{s}^{-1} \mu \mathrm{M}^{-1}\right]}\end{array}$ & $\begin{array}{l}\mathrm{IC}_{50} \\
\text { (- Enz) } \\
{[\mathrm{nM}]}\end{array}$ & $\begin{array}{l}\mathrm{IC}_{50} \\
(+ \\
\text { Enz) } \\
\text { [nM] }\end{array}$ & $\mathrm{QIC}_{50}$ \\
\hline $\begin{array}{l}\text { reso- } \\
\text { GIcA }\end{array}$ & $36 \pm 8$ & $14.2 \pm 2.3$ & $0.39 \pm 0.02$ & - & & - \\
\hline 9 & $33 \pm 5$ & $20.7 \pm 2.4$ & $0.62 \pm 0.02$ & - & & - \\
\hline 10 & $3.6 \pm 0.2$ & $4.7 \pm 0.1$ & $1.31 \pm 0.04$ & - & & - \\
\hline $\begin{array}{l}\text { SN-38- } \\
\text { GlcA }\end{array}$ & - & - & - & 1528 & 15.2 & 100.5 \\
\hline 11 & $42 \pm 4$ & $263 \pm 14$ & $6.26 \pm 0.30$ & 642.1 & 58.7 & 10.9 \\
\hline 12 & $50 \pm 5$ & $273 \pm 14$ & $5.45 \pm 0.25$ & 1921 & 81.9 & 23.4 \\
\hline
\end{tabular}

high yield on the same precursor molecule, whereas drug conjugation is performed via less demanding, readily available syntheses. Extended scaffold glucuronides undergo enzymatic bioconversion with kinetics parameters similar or even favorable when compared to the bioconversion of native glucuronides. In cell culture, extended scaffold glucuronides reversibly mask toxicity of the incorporated toxin, as is used in the enzyme prodrug therapies for localized drug synthesis. ${ }^{5}$ This strategy to glucuronide prodrug design is directly applicable to a wide range of phenols varied by their $\mathrm{pKa}$ and includes tyrosine, towards glucuronidation of peptides, and is readily extendable to other functionalities common in drugs such as amines (as is already realized in e.g. Brentuximab Vedotin) and aliphatic hydroxyls.

ACKNOWLEDGMENTS. ANZ acknowledges funding from the European Research Council (ERC-2013-CoG 617336 BTVI). T. Poulsen Lab (Aarhus University) is acknowledged for access to a preparative HPLC and Dr. T. Breitenbach for providing us with the HeLa cells.

\section{REFERENCES}

1. P. J. Carter and G. A. Lazar, Nat. Rev. Drug Discov., 2017, 17, 197.

2. T. Rodrigues and G. J. L. Bernardes, Angew. Chem. Int. Ed., 2018, 57, 2032-2034.

3. Z. M. Prijovich, P.-A. Burnouf, H.-C. Chou, P.-T. Huang, K.-C. Chen, T.-L. Cheng, Y.-L. Leu and S. R. Roffler, Mol. Pharma., 2016, 13, 1242-1250.

4. R. Walther, J. Rautio and A. N. Zelikin, Adv. Drug Deliv. Rev., 2017, 118, 65-77.

5. B. Stadler and A. N. Zelikin, Adv. Drug Deliv. Rev., 2017, 118, 1.

6. B. Renoux, F. Raes, T. Legigan, E. Peraudeau, B. Eddhif, P. Poinot, I. Tranoy-Opalinski, J. Alsarraf, O. Koniev, S. Kolodych, S. Lerondel, A. Le Pape, J. Clarhaut and S. Papot, Chem. Sci., 2017, 8, 3427-3433.

7. A. V. Stachulski, G. N. Jenkins, Nat. Prod. Rep., 1998, 15, 173-186.

8. A. V. Stachulski, X. Meng, Nat. Prod. Rep., 2013, 30, 806-848.

9. K. J. Jensen, J. Chem. Soc., Perkin Trans. 1, 2002, 2219-2233.

10. M. Jacobsson, J. Malmberg and U. Ellervik, Carbohydr. Res., 2006, 341, 1266-1281.
11. A. Alouane, R. Labruère, T. Le Saux, F. Schmidt and L. Jullien, Angew. Chem. Int. Ed., 2015, 54, 7492-7509.

12. W. Koenigs and E. Knorr, Chem. Ber., 1901, 34, 957-981.

13. B. Helferich and E. Schmitz-Hillebrecht, Chem. Ber., 1933, 66, 378-383.

14. R. R. Schmidt and J. Michel, Angew. Chem. Int. Ed., 1980, 19, 731732.

15. R. R. Schmidt and J. Michel, Tetrahedron Lett., 1984, 25, 821-824.

16. S. Weck and T. Opatz, Synthesis, 2010, 2010, 2393-2398.

17. C. Jaramillo and S. Knapp, Synthesis, 1994, 1994, 1-20.

18. F. Kratz, I. A. Müller, C. Ryppa and A. Warnecke, ChemMedChem, 2008, 3, 20-53.

19. C. F. Riber, A. A. A. Smith and A. N. Zelikin, Adv. Healthcare Mater, 2015, 4, 1887-1890.

20. P. L. Carl, P. K. Chakravarty and J. A. Katzenellenbogen, J. Med. Chem., 1981, 24, 479-480.

21. T. Sun, A. Morger, B. Castagner and J.-C. Leroux, Chem. Commun., 2015, 51, 5721-5724.

22. P. J. McEnaney, C. G. Parker, A. X. Zhang and D. A. Spiegel, ACS Chem. Biol., 2012, 7, 1139-1151.

23. R. P. Lyon, T. D. Bovee, S. O. Doronina, P. J. Burke, J. H. Hunter, H. D. Neff-LaFord, M. Jonas, M. E. Anderson, J. R. Setter and P. D. Senter, Nat. Biotechnol., 2015, 33, 733.

24. R. V. Kolakowski, K. T. Haelsig, K. K. Emmerton, C. I. Leiske, J. B. Miyamoto, J. H. Cochran, R. P. Lyon, P. D. Senter and S. C. Jeffrey, Angew. Chem. Int. Ed., 2016, 55, 7948-7951.

25. T. Tsunoda, Y. Yamamiya and S. Itô, Tetrahedron Lett., 1993, 34, 1639-1642.

26. Y.-L. Leu, C.-S. Chen, Y.-J. Wu and J.-W. Chern, J. Med. Chem., 2008, 51, 1740-1746.

27. K. Inoue and K. Sakai, Tetrahedron Lett., 1977, 18, 4063-4066.

28. T. Duo, K. Robinson, I. R. Greig, H.-M. Chen, B. O. Patrick and S. G. Withers, J. Am. Chem. Soc., 2017, 139, 15994-15999.

29. J. B. Kempton and S. G. Withers, Biochemistry, 1992, 31, 99619969.

30. Detailed procedures for syntheses and characterization (NMR, HR-MS, HPLC; quantification of optical purity of the prodrugs was beyond the scope of this study) are available in the electronic supplementary information. 